Author Pre-Print - Journal of Applied Physics - May 2014Journal of Applied Physics

115(20):204905-204905-8 DOI: 10.1063/1.4875876

\title{
Modelling of ultrasonic propagation in turbulent liquid sodium with temperature gradient
}

\author{
N. Massacret, ${ }^{1,2}$ J. Moysan, ${ }^{2 a}$ M.A. Ploix, ${ }^{2}$ J.P. Jeannot, ${ }^{1}$ and G. Corneloup ${ }^{2}$
}

${ }^{I}$ CEA, DEN, Nuclear Technology Department, F-13108 Saint-Paul-Lez-Durance, France

${ }^{2}$ Aix-Marseille Université, LMA UPR 7051 CNRS, site LCND, 13625 Aix-en-Provence, France

The use of ultrasonic instrumentation in sodium-cooled fast reactors (SFR) requires to understand and to predict how ultrasonic waves can be deflected, slowed down or speeded up, depending on the thermo-hydraulic characteristics of the liquid sodium. These thermo-hydraulic characteristics are mainly the local temperature and flow speed of the sodium. In this study we show that ray theory can be used to simulate ultrasonic propagation in a medium similar to the core of a sodium-cooled fast reactor, in order to study ultrasonic instrumentation and prepare it installation and utilisation in the sodium of the nuclear reactor. A suitable model has been developed and a set of thermo-hydraulics data has been created, taking account of the particularities of the sodium flow. The results of these simulations are then analysed within the framework of acoustic thermometry, in order to determine which disturbance must be taken into account for the correct operation of the temperature measurement.

\section{INTRODUCTION}

Sodium-cooled fast reactors have been chosen by France as the reference option in the framework of the Generation IV international forum. These reactors must achieve the Generation IV objectives in terms of sustainable development, economic competitiveness, reliability, proliferation resistance and protection of people ${ }^{1}$. The CEA (atomic energy and alternative energies commission), EDF (Electricite de France) and AREVA have formed a partnership to coordinate their research programmes and to develop a fourth generation nuclear reactor prototype.

One of the many developments necessary to reach the industrial standard is a research programme named ISIR (In-Service Inspection and Repair) which has been initiated by the three partners ${ }^{2}$. One of the objectives of this research programme is to take the configuration of this new reactor and the characteristics of the coolant into account in order to develop suitable instrumentation for continuous in-service monitoring. Actually in these reactors, the coolant is liquid sodium which is an opaque liquid metal, preventing the use of conventional optical methods and reaching temperatures around $550^{\circ} \mathrm{C}$ in normal operating. Various instrumentation techniques

\footnotetext{
a : Author to whom correspondence should be addressed. Electronic mail: joseph.moysan@univ-amu.fr
} 
Author Pre-Print - Journal of Applied Physics - May 2014Journal of Applied Physics 115(20):204905-204905-8 DOI: 10.1063/1.4875876

associated with the use of sodium are studied in this programme, including detection of bubbles in the sodium, ultrasound transmission through interfaces, and acoustic thermometry $3,4,5$.

Acoustic thermometry is a technique based on the temperature-dependent speed of ultrasonic waves in a medium. It is possible to propagate the ultrasounds in a solid wave-guide immersed in the hot fluid, as in the case of some acoustic thermometers ${ }^{6}$, or propagate them directly in the fluid: it is this second application that is under consideration in this paper. High temperature transducers are also studied ${ }^{7,8,9}$. We plan to develop a method involving the propagation of an ultrasonic wave to two surfaces, separated by known distance, which generate echoes. The mean local speed of the medium is obtained by measuring the ultrasonic wave time of flying between these two surfaces. As the relationship between the speed and the temperature is known, the temperature of the sodium is obtained. Leibowitz et al. have established a relationship between ultrasound speed and temperature in sodium $^{10}$ :

$$
c_{u s}=2577.3-0.536 \cdot T
$$

where $c_{u s}$ is the speed of the ultrasonic waves in meters per second and $T$ is the temperature of the sodium in degrees Celsius. This formula is valid between $182^{\circ} \mathrm{C}$ and $896^{\circ} \mathrm{C}$ with an accuracy of $0.4 \%$ in pure sodium.

For in-reactor application, the edges of the fuel subassembly heads are good candidates for generating the echoes. It would then be possible to measure the temperature of the sodium directly on the diameter of the subassembly outlets. An example of an experimental configuration is shown in figure 1.

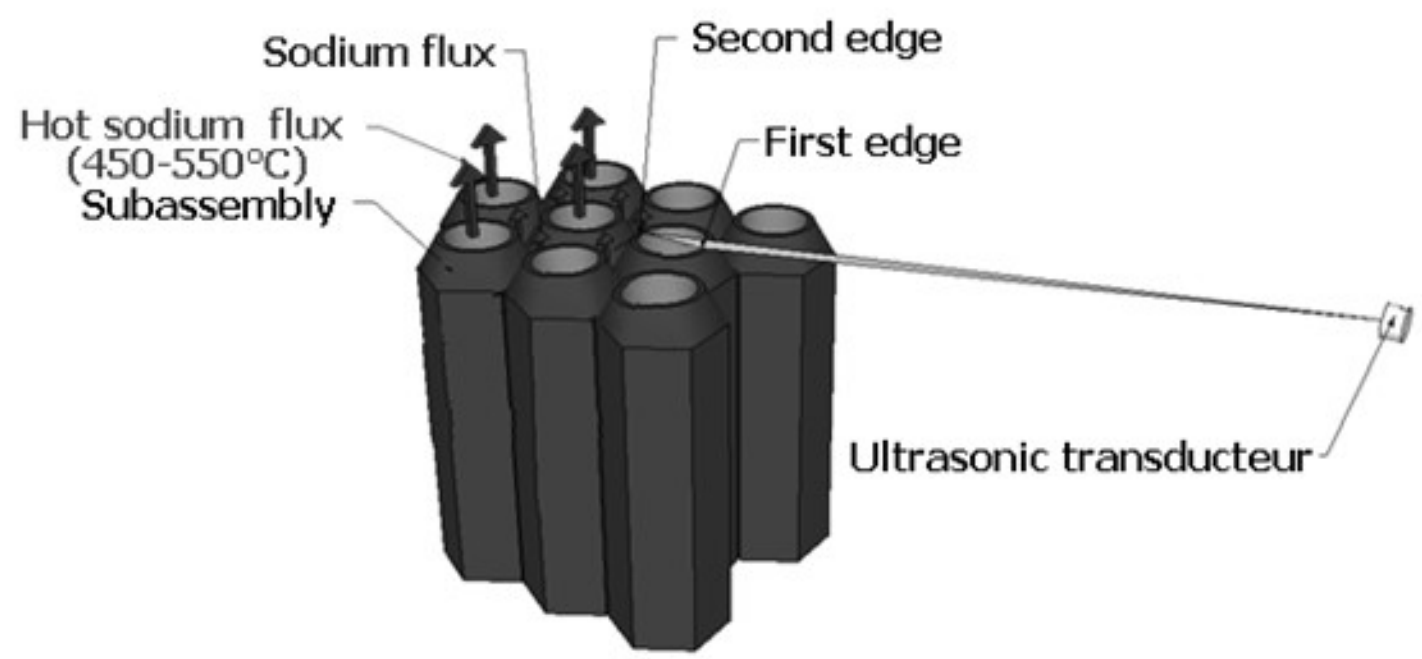

FIG. 1. Acoustic thermometry principle with a grazing incidence beam. 
Author Pre-Print - Journal of Applied Physics - May 2014Journal of Applied Physics 115(20):204905-204905-8 DOI: 10.1063/1.4875876

This method constitutes the subject of a patent by the UKAEA (United Kingdom Atomic Energy Authority) in $1985^{11}$. It is possible to obtain excellent measurement dynamics: as the speed of ultrasonic waves is around 2300 $\mathrm{m} . \mathrm{s}^{-1}$ in sodium at $550^{\circ} \mathrm{C}$, the response time is mainly due to the signal processing time. Moreover it would be possible, with only one transducer, to measure the temperature of the sodium exiting several subassemblies. This was proved in water experiment, where the temperature of the fluid exiting a row of six subassemblies was measured, using a single transducer emitting a grazing incidence beam ${ }^{12}$. This method would significantly reduce the amount of instrumentation above the core.

However the thermo-hydraulic conditions above the core may disturb the propagation of the ultrasonic waves between the ultrasonic transducer and the subassembly heads, in terms of time delay and deflection.

There are several sources of thermal inhomogeneities above the core: the temperature difference of the sodium flowing out two neighbouring subassemblies could reach $50^{\circ} \mathrm{C}$ following the core conception. Moreover the sodium circulating in the spaces between the subassemblies, and the one flowing out the spaces left clear for insertion of control rods or safety devices, are cooler by several tens of degrees Celsius, than the sodium flowing out the subassemblies. The ultrasonic waves will therefore propagate in a medium in which the temperature is very inhomogeneous.

In addition, the flow above the core is turbulent with local speeds reaching approximately $3 \mathrm{~m} \cdot \mathrm{s}^{-1}$, and speed gradients are about several metres per second per centimetre. This turbulent field may also have consequences on the propagation of the ultrasonic waves. This phenomena is used in acoustic debitmeter to measure the speed flow $^{13-14}$. In the case of acoustic thermometry, it could lead to errors in the measurement of the temperature.

In order to be able to anticipate and deal with these potential problems, a $2 \mathrm{D}$ ultrasonic propagation model, suitable for this medium and this instrumentation, has been studied to simulate the path of the ultrasonic waves. These simulations will enable the disturbance caused by the thermo-hydraulic characteristics of sodium to be quantified, and will determine of whether they are problematic for acoustic thermometry. In this article, the reasons for choosing this model will be explained, then its theory detailed. Finally, various simulations highlighting probable types of disturbance and their effects on acoustic thermometry will be analysed.

\section{THE ACOUSTIC MODEL}

\section{A. Choice of the appropriate model}


Author Pre-Print - Journal of Applied Physics - May 2014Journal of Applied Physics 115(20):204905-204905-8 DOI: 10.1063/1.4875876

It is important to take the thermo-hydraulic characteristics of the medium into account when choosing an appropriate, effective ultrasonic propagation model. Some codes have been developed for extremely turbulent media in which the Mach numbers are close to one, using methods involving different degrees of parabolic approximation ${ }^{15}$. Despite the precision of these codes, the sodium flow above the core of an SFR is not fast enough in comparison with the speed of ultrasonic waves to justify the use of such complex methods. For this study, in which the Mach number is around $10^{-3}$, it is possible to use the frozen fluids hypothesis, in which the thermohydraulic conditions of the medium are considered to be stationary when an ultrasonic wave is propagated. This approximation is used in particular for ultrasonic studies of turbulence in fluid media ${ }^{16}$.

Another important parameter is the ratio between the characteristic size of the inhomogeneities of the medium and the ultrasonic wavelength. The size of the thermal inhomogeneities depends mainly on the thermal conductivity of sodium. As it is a liquid metal, its thermal conductivity is high and its Prandtl number is very low, around $10^{-3}$. The Prandtl number is the ratio between kinematic viscosity and thermal diffusivity. It is used to determine whether the temperature field of a fluid will be significantly influenced by its speed field (high Prandtl number), or influenced by it very little (low Prandtl number). Small thermal inhomogeneities will therefore disappear quickly in this medium. Several experiments have been carried out in sodium, and it seems that the characteristic size of the thermal inhomogeneities is more than 2 centimetres ${ }^{17-18}$. The velocity of a $2.25 \mathrm{MHz}$ ultrasonic wave propagating in sodium at $550^{\circ} \mathrm{C}$ being around $2300 \mathrm{~m} \cdot \mathrm{s}^{-1}$, its wavelength is around a millimetre. It is therefore possible to make the assumption of high frequency waves ${ }^{14}$ :

$$
\lambda \ll L \text { and } \sqrt{\lambda X} \ll L
$$

where $\lambda$ is the ultrasonic wavelength, $L$ the characteristic size of the thermal inhomogeneities, and $X$ the length of the path travelled by the ultrasonic waves in the fluid.

With these two assumptions (frozen fluid and high frequency), the acoustic ray model based on geometric acoustic theory must be favoured ${ }^{14}$.

Another important parameter which encourages the choice of this model is the calculation cost. A large number of simulation codes use the finite or spectral element method ${ }^{19-22}$. These codes are very efficient for simulating the interference of an ultrasonic wave with inhomogeneities and the distribution of the acoustic amplitude in the medium. However, calculating the propagation of a wave of several megahertz over several tens of centimetres generates significant calculation costs. 
Author Pre-Print - Journal of Applied Physics - May 2014Journal of Applied Physics

115(20):204905-204905-8 DOI: 10.1063/1.4875876

The application of the ray tracing method request generally few numerical resources because the calculations concern only the small part of the geometry in the area of the propagating ray. Moreover the calculation of the path of each ray is independent, so it is possible to calculate these paths in parallel on several processors. In order to determine the distribution of the acoustic amplitude, it is then possible to apply the Gaussian beam summation method.

\section{B. Acoustic ray model}

The geometric acoustic ray model consists on following the spatial progress of a point on a wavefront. The path travelled by this point is called the acoustic ray. A wavefront is defined by all the particles in the propagation medium which, at a given time, are set in motion by the passing of an alternation. An iterative method is used in this study: at each step of the calculation, the local thermo-hydraulic characteristics (temperature and flow speed of the sodium) are used to calculate the new direction of propagation of the wave. The path of the ray is then calculated according to this direction over a given distance. The same operation is carried out at this new position. Thus, the two most important steps at each iteration, are taking the thermo-hydraulic data into account and calculating the direction of propagation.

The calculation model is based on the fact that if a particle in the medium is moving at a flow speed $\vec{v}(\vec{x})$, the wave will propagate at a speed $\vec{v}+c \cdot \vec{N}$, where $\vec{N}$ is the local direction of propagation of the wave (i.e. normal to the wavefront) and $c$ is the velocity of the ultrasonic waves in an equivalent static fluid ${ }^{23}$. With $\vec{x}_{p}(t)$ a point on the wavefront, this point moves at speed $\vec{v}+c \cdot \vec{N}$, thus:

$$
\frac{d \vec{x}_{p}}{d t}=\vec{v}\left(\vec{x}_{p}, t\right)+\vec{N}\left(\vec{x}_{p}, t\right) \cdot c\left(\vec{x}_{p}, t\right)
$$

Then let $\tau$ is the function describing the coordinates of each point of the wavefront. At instant $t$, all the points $\vec{x}_{p}$ belong to the wavefront and are described by this function. Thus:

$$
t=\tau\left(\vec{x}_{p}\right)
$$

If the time interval $\Delta t$ between two positions on the wavefront is short enough, it is possible to write:

$$
t+\Delta t \approx \tau\left(\vec{x}_{p}(t)+\dot{\vec{x}}_{p}(t) \Delta t\right) \approx \tau\left(\vec{x}_{p}\right)+\Delta \mathrm{t} \dot{\vec{x}}_{p}(t) \cdot \vec{\nabla} \tau
$$


Author Pre-Print - Journal of Applied Physics - May 2014Journal of Applied Physics 115(20):204905-204905-8 DOI: 10.1063/1.4875876

I.e. $\vec{\nabla} \tau=\vec{s}$, with $\vec{s}$ is the slowness vector:

$$
\vec{s}=\frac{\vec{N}}{c+\vec{v} \cdot \vec{N}}
$$

Since $\vec{s}$ is dependent on $\vec{x}_{p}(t)$ :

$$
\frac{d \vec{s}\left(\vec{x}_{p}\right)}{d t}=\vec{\nabla} \vec{s} \cdot \dot{\vec{x}}_{p}=c(\vec{N} \cdot \vec{\nabla}) \vec{s}+(\vec{v} \cdot \vec{\nabla}) \vec{s}
$$

And:

$$
\begin{aligned}
& \frac{d \vec{s}\left(\vec{x}_{p}\right)}{d t}=\frac{c}{\Omega}(\vec{s} \cdot \vec{\nabla}) \vec{s}+(\vec{v} \cdot \vec{\nabla}) \vec{s} \\
& \text { With } \Omega=\frac{c}{c+\vec{v} \cdot \vec{N}}=1-\vec{v} \cdot \vec{s}
\end{aligned}
$$

It is possible to define the location of any point $\vec{x}_{p}(t)$ on the wavefront with a system using two equations:

$$
\left\{\begin{array}{c}
\frac{d \vec{x}_{p}}{d t}=\vec{v}\left(\vec{x}_{p}, t\right)+\frac{c^{2}\left(\vec{x}_{p}, t\right)}{\Omega} \vec{s} \\
\frac{d \vec{s}\left(\vec{x}_{p}\right)}{d t}=-\frac{\Omega}{c} \vec{\nabla} c-\vec{s} \wedge(\vec{\nabla} \wedge \vec{v})-(\vec{s} \cdot \vec{\nabla}) \vec{v}
\end{array}\right.
$$

Thus, using these two equations (9), the path of an acoustic ray can be determined.

\section{Gaussian beam summation}

Ray theory cannot be used on its own to determine the amplitude of the acoustic signal at all the points of the geometry. This amplitude is considered to be zero out of the rays, which is not the physical reality.

To overcome this drawback the Gaussian beam summation method can be applied ${ }^{14,24-26}$. The first step in this method involves calculating the propagation of a beam of acoustic rays. Each beam is then given a Gaussian amplitude profile. This profile is characterised by a Gaussian decay according to the distance from the ray. Its width at mid-height and its maximum amplitude are two parameters which change along the ray according to the frequency of the propagated wave. Then, at the point at which it is necessary to know the acoustic amplitude of the signal, the amplitude from each ray is summed. With this Gaussian decay of the energy, the further a ray is from the point of interest, the weaker its energy contribution at this point. 
Author Pre-Print - Journal of Applied Physics - May 2014Journal of Applied Physics 115(20):204905-204905-8 DOI: 10.1063/1.4875876

Again, the advantage of this method is its speed: it is not necessary to apply it along the whole length of the acoustic beam to obtain the amplitude at a specific point; it simply needs to be applied in the area concerned and at a chosen moment.

The starting point of the calculation is the conventional wave equation, here in a 2 -dimensional medium ${ }^{24}$ :

$$
\frac{\partial^{2} \mathrm{u}}{\partial \mathrm{x}^{2}}+\frac{\partial^{2} \mathrm{u}}{\partial \mathrm{y}^{2}}=\frac{1}{\mathrm{c}^{2}} \frac{\left(\partial^{2} \mathrm{u}\right)}{\partial \mathrm{t}^{2}}
$$

where $u$ is a physical parameter linked to the movement of the wave, $c(x, y)$ is the speed of the wave and $t$ is the time.

The first step in the demonstration is to change the system of coordinates: the Cartesian coordinates system (here $x$ and $y$ ) is abandoned in favour of the coordinates linked to the ray in question. Giving $\vec{t}$ the direction vector which is tangent to the ray, and $\vec{n}$ the direction vector which is orthogonal to it. The scheme in FIG 2. Coordinate system linked to the ray.

explains this change of coordinates.

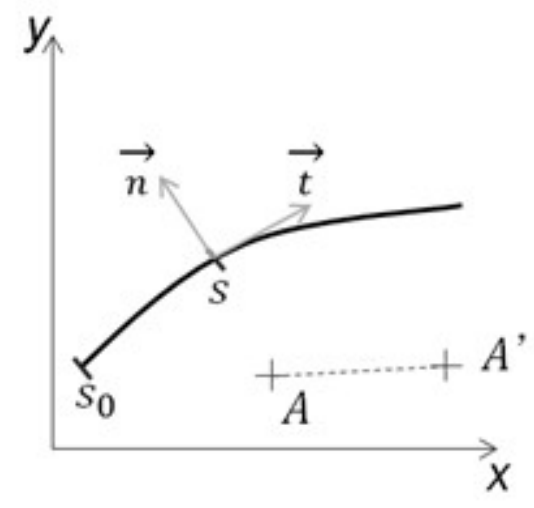

FIG 2. Coordinate system linked to the ray.

The scalar $s$ also appears in this diagram. This indicates the curvilinear abscissa of the ray. $s$ is incremented at each iteration when the ray method is applied. Point A is a point of interest in the medium and A' is the position of point A following an iteration. In the coordinates system $(s, n)$ the wave equation $(10)$ becomes:

$$
\frac{1}{h} u_{, s s}+h u_{, n n}-\frac{h}{c^{2}} u_{, t t}+u_{, s}\left(\frac{1}{h}\right)_{s}+u_{, n} h_{, n}=0
$$


Author Pre-Print - Journal of Applied Physics - May 2014Journal of Applied Physics

115(20):204905-204905-8 DOI: 10.1063/1.4875876

With: $h=\frac{c(s, n)}{c(s, 0)}$

For formula (11), the partial derivatives are indicated using a comma. This notation will be used in the remainder of this article.

Then the parabolic approximation method is applied. This method enables only those waves propagating in a small aperture cone centred on the axis of the ray to be taken into account in the calculation. Waves created during reflection or diffraction are thus ignored. This method reduces the calculation cost substantially. It is also fully justifiable here, as this study is only examining the propagation of the waves: interactions with the solid structures in the medium are not being studied. In addition, since the assumption of high frequency waves is applied, the medium in which the calculations are carried out does not contain any thermo-hydraulic phenomena which could cause diffraction or diffusion.

The application of this method consists of stating $u$ in the form:

$$
u(s, n, t)=U(s, n, w) \cdot \exp \left\{-i w\left[t-\int_{s_{0}}^{s} \frac{d s}{c(s)}\right]\right\}
$$

Where $t$ is the time, $w$ is the angular frequency of the signal and $U$ is a constant of the same type as $u$, specific to each ray.

A condition is thus placed on $n$ in the steps of the calculation:

$$
n=0\left(w^{-\frac{1}{2}}\right)
$$

Where the value $1 / 2$ has been defined empirically ${ }^{27}$. The notation 0 means that $n$ wouldn't be much more superior to

$w^{-\frac{1}{2}}$ and therefore that the scope of the method is only suitable for an area close to the ray, which decreases as the angular frequency increases.

Following the resolution steps of Cerveny ${ }^{24}$, a system of two linear differential equations is introduced by inserting two complex functions $p(s)$ and $q(s)$, and a real value $\Phi$ which can differ from one ray to another. The amplitude of the acoustic signal $u(s, n, t)$ at a certain distance $n$ from the ray is thus calculated using a three-equation system:

$$
\left\{\begin{array}{c}
q_{, s}=c p \\
p_{, s}=-\frac{q}{c^{2}} c, n n \\
u(s, n, t)=\Phi\left[\frac{c(s)}{q(s)}\right]^{\frac{1}{2}} \exp \left[-i \omega\left(t-\int_{s_{0}}^{s} \frac{d s}{c(s)}\right)+\frac{i \omega}{2} \frac{p}{q} n^{2}\right]
\end{array}\right.
$$


Author Pre-Print - Journal of Applied Physics - May 2014Journal of Applied Physics 115(20):204905-204905-8 DOI: 10.1063/1.4875876

Finally the contributions of each ray at a particular point in the simulated medium must be summed in order to ascertain the acoustic energy at this point. In order to be able to observe the changes in a wavefront, or carry out a simulation with an acoustic signal as close as possible to reality, this code has been implemented so as to give $\Phi$ a real value that can be time-dependent. For the following simulations, in order to simplify the results, a very simple signal is used: several alternations of $2.25 \mathrm{MHz}$ frequency waves. Its time signal is shown in FIG 3.

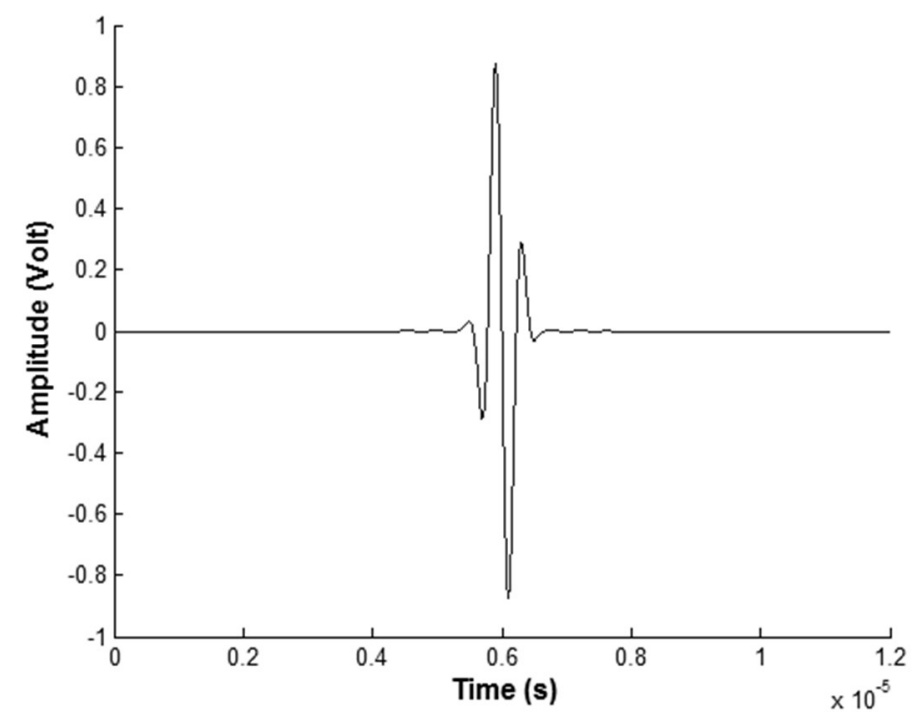

FIG 3. Simple signal with a wave at $2.25 \mathrm{MHz}$.

\section{THERMO-HYDRAULIC DATA FOR THE SIMULATED MEDIUM}

\section{A. Nature of the data}

To preserve the speed of the code, it has been necessary to handle the thermo-hydraulic data in a specific way. The frequency of the waves propagated in this code is in the region of a few megahertz. To simulate their propagation correctly, it is recommended that for each calculating step a ray is propagated on a distance of $\frac{c_{\min }}{2 \pi f}$, i.e. a distance close to a tenth of a millimetre ${ }^{28}$. In addition, as the path of a ray is sensitive to variations in the thermohydraulic conditions of the medium, it is not possible to propagate it in a coarse set of thermo-hydraulic data in which these conditions vary suddenly from one area to another with the risk of no longer complying with the assumption of high frequency waves (see Section 2.A.).

It is possible to use a set of thermo-hydraulic data containing grids close to a tenth of a millimetre, but this will generate a huge amount of data and lots of these will be unnecessary. In fact the characteristic lengths of the 
Author Pre-Print - Journal of Applied Physics - May 2014Journal of Applied Physics

115(20):204905-204905-8 DOI: 10.1063/1.4875876

inhomogeneities (temperature or speed) which occur in this medium are around a centimetre and there would therefore be significant redundancy of data ${ }^{29}$.

It was therefore decided to carry out a linear interpolation of the thermo-hydraulic data to determine the temperature and speed conditions in the location through which the ray passes. In this way, it is possible to use very varied sets of thermo-hydraulic data (with fine or coarse grids depending on the turbulence of the medium), while maintaining temperature or flow speed variations descriptions that are fine enough to allow propagation of the ray. Moreover it is possible to use results of thermo-hydraulics simulations with various meshing (structured or not). This is an interesting point for research gathering thermo-hydraulics and acoustics issues.

In the following first simulations given in this article, digitally generated sets of thermo-hydraulic data will be used. Thus we propose a simplified model which reproduced the reality in terms of size and amplitude of the inhomogeneities.

\section{B. Simulated media}

As shown previously, the thermo-hydraulic conditions of the medium will influence the propagation of ultrasonic waves in liquid sodium. It is therefore important to know to what extent this disturbance will occur and if it will affect ultrasonic propagation significantly. Three simple cases are simulated in this article, with the aim of studying the temperature and flow effects separately. The medium is the liquid sodium just above the subassembly heads.

When carrying out acoustic measurement of the temperature of the sodium at the outlet of a subassembly, an ultrasonic beam propagates above the core until it is reflected on the edges of the subassembly. This beam successively encounters hot areas and cold areas, created respectively by the sodium exiting the subassemblies and the spaces between the subassemblies. The shape of the sodium thermal and flow inhomogeneities will disturb the ultrasonic waves to a greater or lesser extent depending on the direction of ultrasonic propagation. This medium also has an inhomogeneous flow speed field map: the hot sodium exiting the subassemblies moves at a speed of around $3 \mathrm{~m} \cdot \mathrm{s}^{-1}$, creating a significant speed gradient with the sodium moving between the subassemblies at a much lower speed (close to $0 \mathrm{~m} \cdot \mathrm{s}^{-1}$ ).

The first medium simulated in this study is an horizontal cross-section of a row of three subassemblies, discharging hot sodium at $570{ }^{\circ} \mathrm{C}$. The temperature of the sodium in the spaces between the subassemblies is 
Author Pre-Print - Journal of Applied Physics - May 2014Journal of Applied Physics 115(20):204905-204905-8 DOI: 10.1063/1.4875876

$500^{\circ} \mathrm{C}$. The thermal gradient is linear and extends over a distance equal to the thickness of one subassembly edge.

A map of the temperatures of this geometry, is shown in figure 4 .

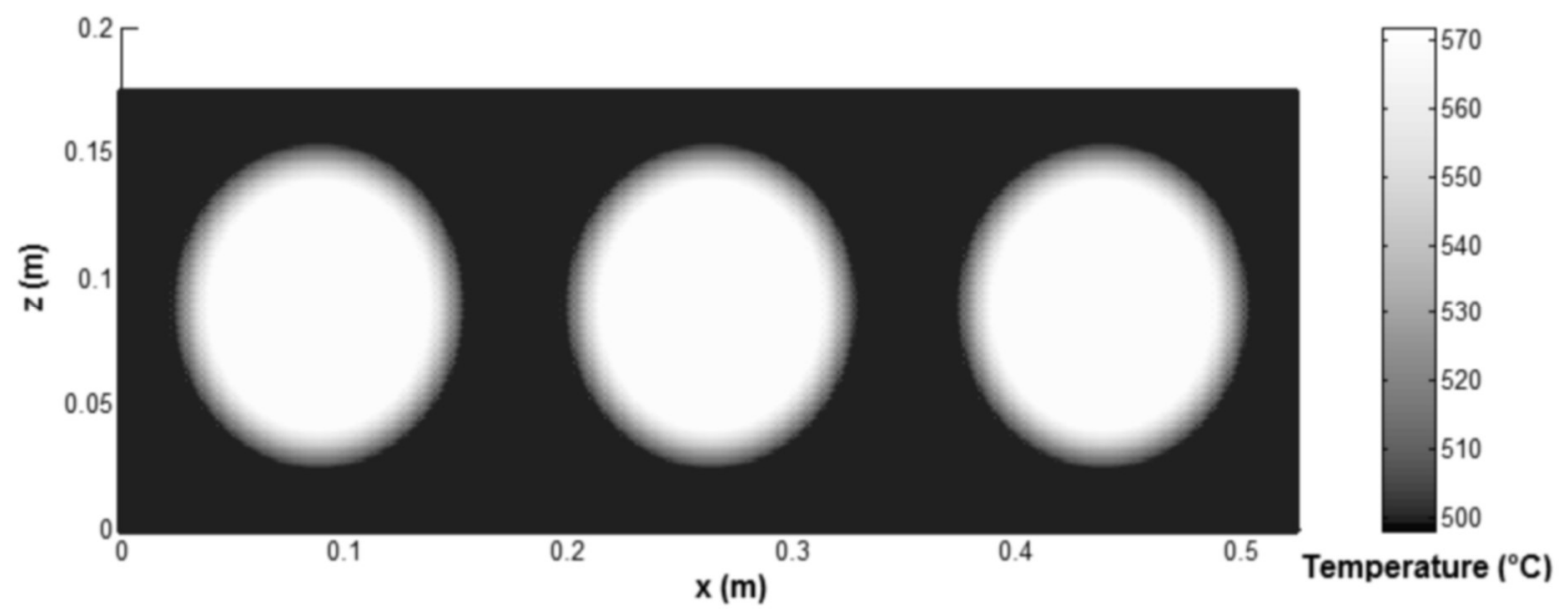

FIG 4. Thermal map of three subassemblies (Case 1).

The flow speed is not taken into account in this case, only the effect of the temperature. The aim here is to highlight the influence of the specific nature of the temperature field just above the subassemblies: in this situation there is no random distribution of the temperature which changes over time, but relatively static temperature gradients. It is thus interesting to observe the accumulation of these organised and repeated inhomogeneities on an ultrasonic beam.

The second geometry used for the simulations will additionally take into account certain subassemblies adjacent to a row of subassemblies on which ultrasonic thermometry is being carried out. The geometry of the second case is shown in FIG 5. 
Author Pre-Print - Journal of Applied Physics - May 2014Journal of Applied Physics

115(20):204905-204905-8 DOI: 10.1063/1.4875876

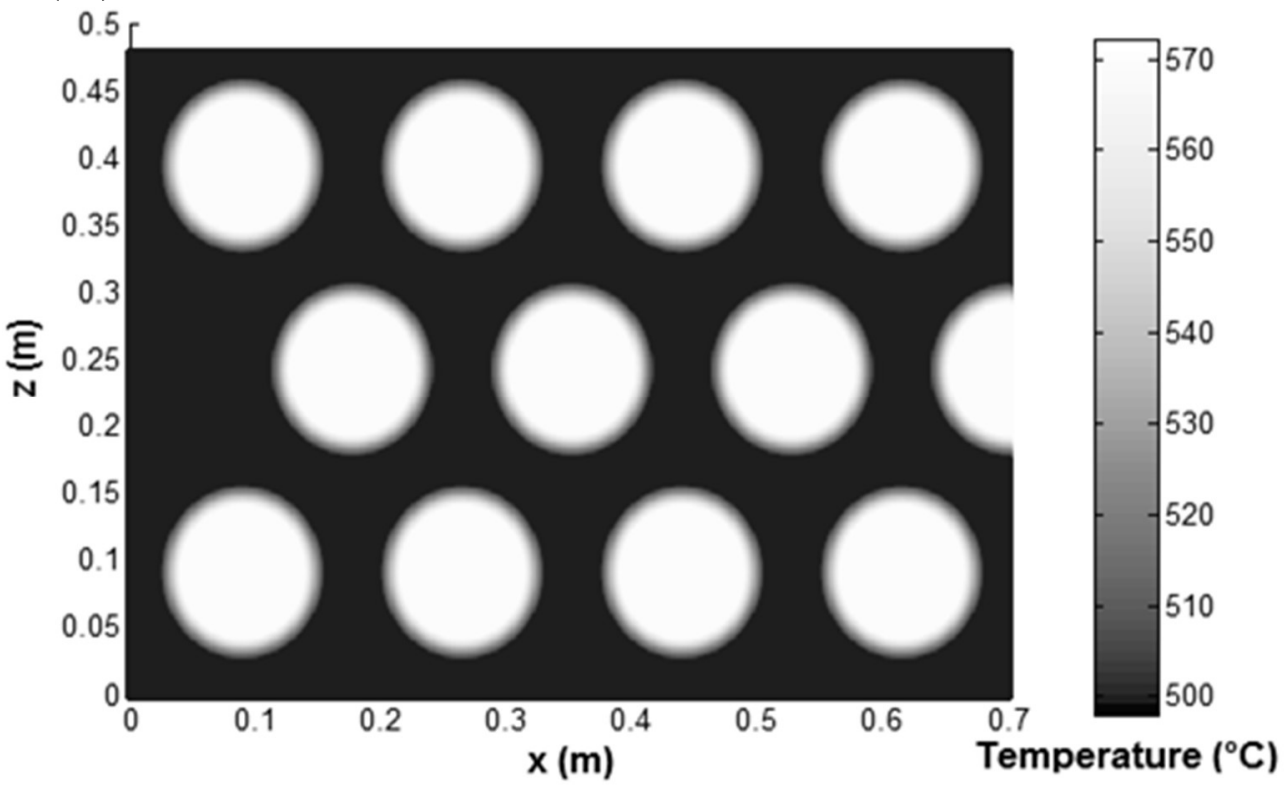

FIG 5. Thermal map of a grid of subassemblies (Case 2).

As previously, the sodium exiting the subassembly heads is at $570^{\circ} \mathrm{C}$ and that in the spaces between the subassemblies is at $500^{\circ} \mathrm{C}$. The thermal inhomogeneities are stable over time and space and the ultrasonic beam will therefore be propagated through a grid of thermal inhomogeneities. Here also the movement of the sodium is not taken into account. The aim of the corresponding simulation is to determine whether a beam propagating through this grid will adopt specific directions of propagation, which could disturb the thermometry of certain subassembly heads.

The third case studied is a vertical cross-section of the speed field of the sodium from three aligned subassemblies. This map is shown in FIG 6.
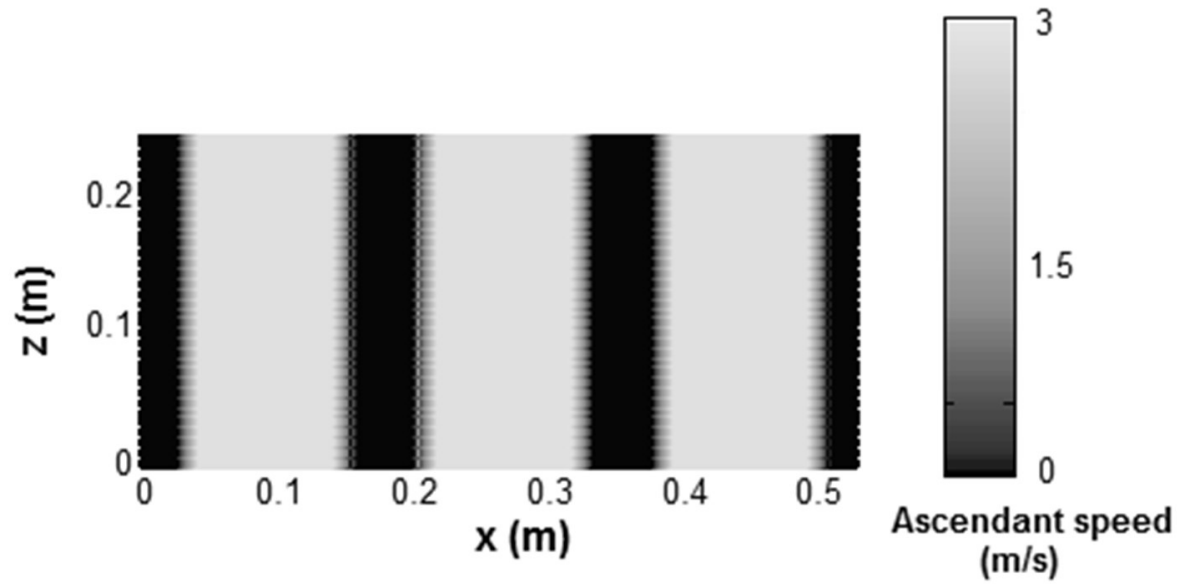

FIG 6. Ascendant speed map of three subassemblies - Vertical cross-section (Case 3). 
Author Pre-Print - Journal of Applied Physics - May 2014Journal of Applied Physics 115(20):204905-204905-8 DOI: 10.1063/1.4875876

This geometry highlights the three ascending vertical flows of sodium exiting each of the three subassemblies. The speed of this flow is $3 \mathrm{~m} \cdot \mathrm{s}^{-1}$. The sodium between the subassemblies is considered to be stationary. In the case of acoustic thermometry, an ultrasonic beam propagates through these flows up to the subassembly heads under consideration. The simulation to be carried out with this geometry does not take into account the effect of the temperature variations nor the turbulent movements which may occur in the mixed layer between the moving and stationary fluids. Its aim is simply to observe the cumulative effect of the repeated strong speed gradients on the path of beams with several incidence angles, and to see whether the effect is problematic for thermometry. The conclusions of these simulations will enable us to determine whether it is necessary to add this effect to that of the temperature in the cases covered in this study, or whether the effect of the temperature alone is adequate to describe wave propagation.

\section{RESULTS AND DISCUSSIONS}

The first simulation below consists in ultrasonic rays propagating along a row of subassemblies (case 1). One hundred parallel rays are generated at an abscissa close to zero to obtain a beam in the axis of the row of subassemblies. The diameter of the beam is $50 \mathrm{~mm}$. The results of this simulation are given in FIG 7.A) and B). 

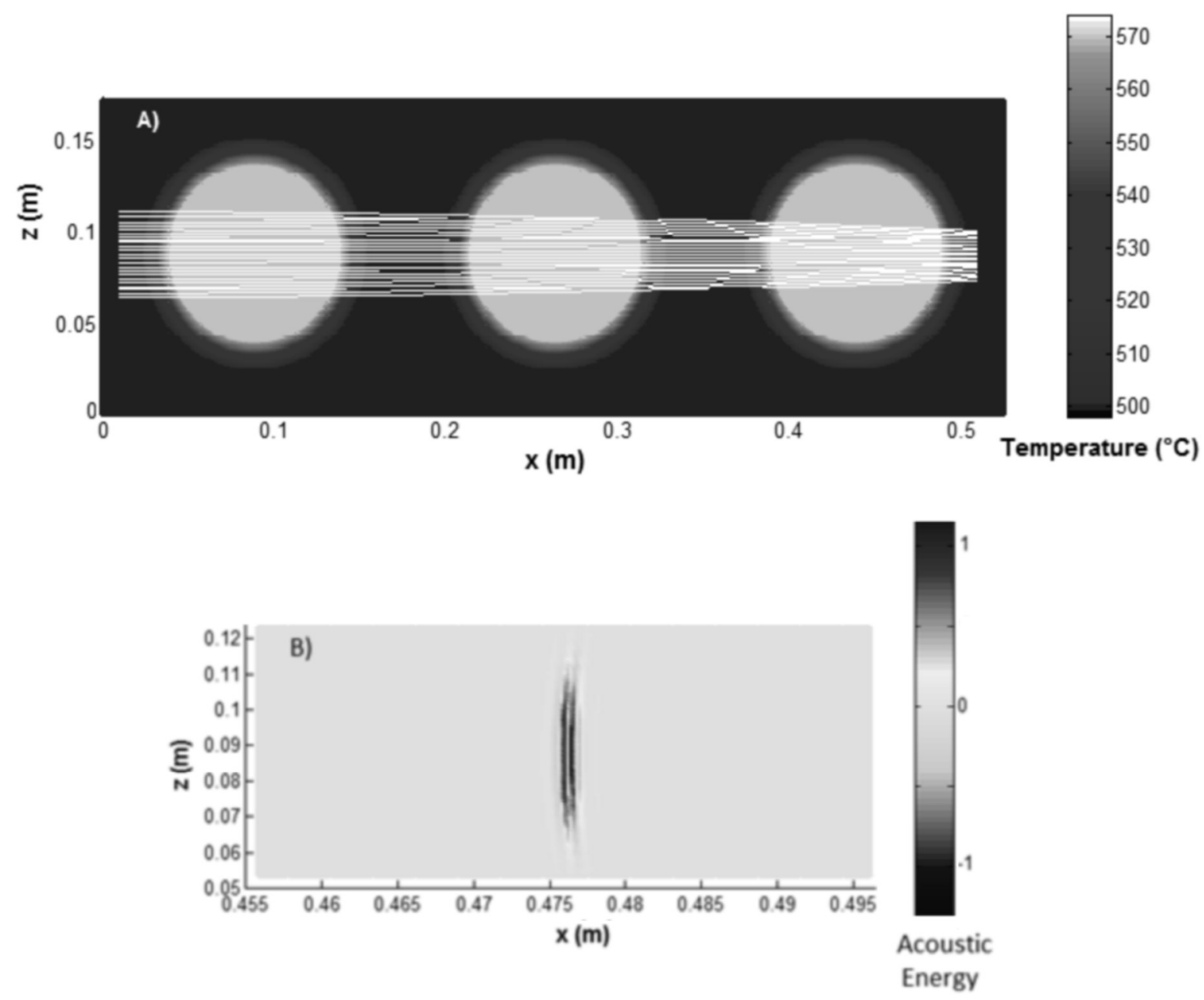

FIG 7. A) Propagation of rays along a row of subassemblies. B) Wavefront obtained with the Gaussian beam summation method.

FIG 7.A shows the path followed by the simulated rays. Ultrasonic deflections appear in the areas in which propagating rays are not collinear with the gradient. This simulation shows that the rays curve towards the centre of the row of subassemblies. This is due to the fact that the ultrasonic waves are deflected towards the areas in which the speed of the medium is the lowest ${ }^{26}$. Here the sodium is hotter at the centre of the subassemblies and according to equation (1), it is in this area that ultrasonic waves propagate most slowly.

FIG 7.B shows the amplitude of the wavefront at a given time calculated with the Gaussian beam summation method. This figure presents the focusing of the beam in axis of the row of subassemblies and makes it possible to see whether the propagation of the rays in various areas in which the speed varies, causes a delay on certain parts of the wavefront. The wavefront shown in FIG 7.B has a slightly concave shape due to the faster propagation of the rays on each side of the beam, but this deformation remains weak. Thus, depending on the shape of the echoes 
Author Pre-Print - Journal of Applied Physics - May 2014Journal of Applied Physics 115(20):204905-204905-8 DOI: 10.1063/1.4875876

returned by the convex and concave edges of a subassembly head, the signal treatment of this ultrasonic beam for ultrasonic thermometry could give a good quality signal.

The simulation with the second set of thermo-hydraulic data studies the situation in which an error has been made on the orientation of the ultrasonic transducer. The simulated rays are emitted at an angle of $5^{\circ}$ in relation to the axis of the row in question, and are slightly offset from it. As previously, these rays are parallel with one another. FIG 8 show the results of this simulation. 

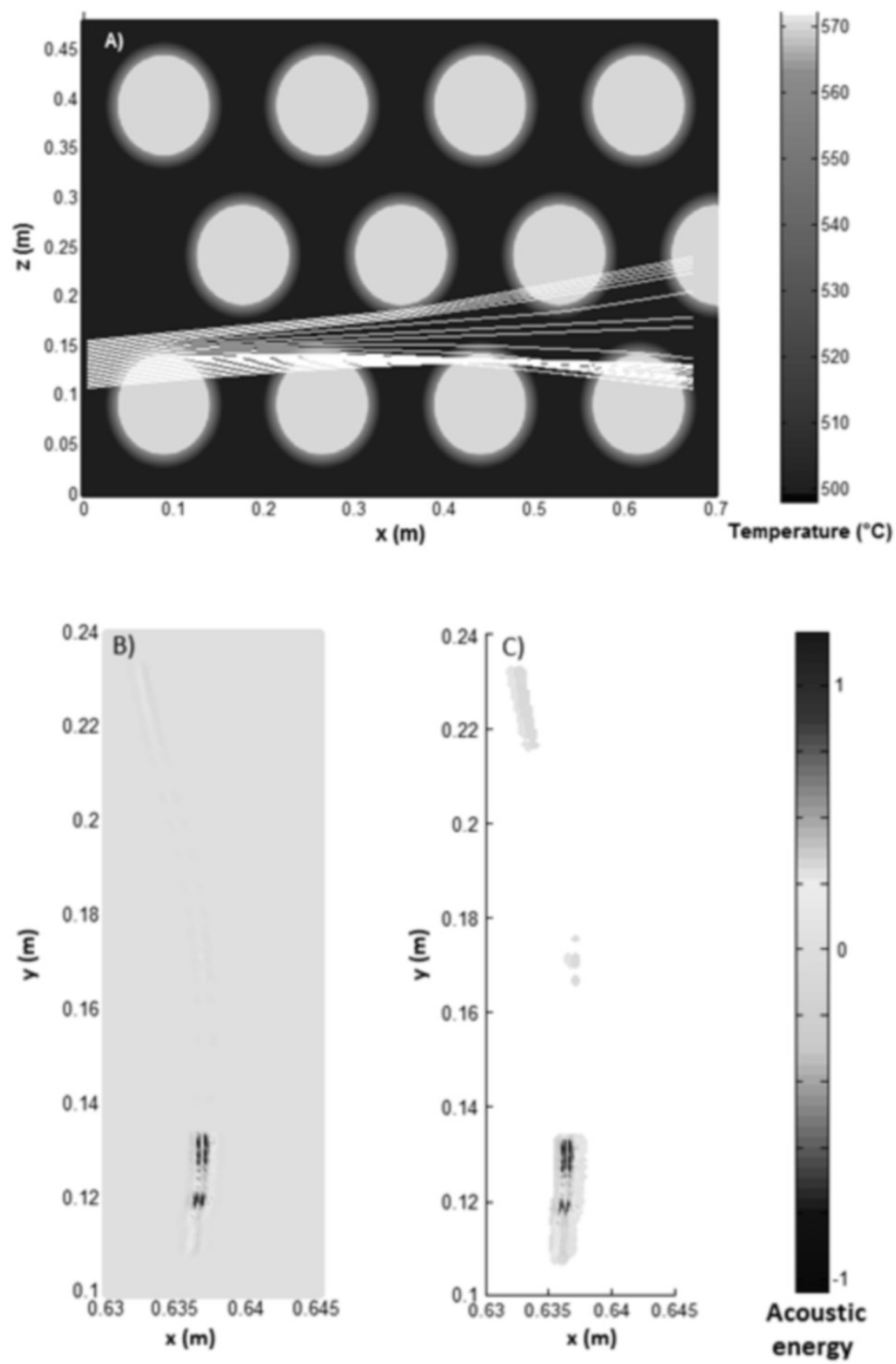

FIG 8. A) Rays propagation among subassemblies. B) and C) Wavefront obtained with the Gaussian beam summation method.

FIG 8.A shows the path followed by each of the rays. The result indicates that for an angle of $5^{\circ}$, most of the rays entering the thermal gradient area of the first row of subassemblies are redirected towards its axis. Likewise, the rays entering the area of influence of the second row are redirected towards its axis. In figure FIG 8.B there are three separate wavefronts. Figure $\mathrm{C}$ enhances results using thresholding. The ultrasonic beam is therefore divided into three parts. Knowing the angle of incidence of the rays with the external and internal edges of the subassembly heads, it is unlikely that echoes returning directly towards the transducer will be generated. The consequence of this orientation error could therefore be the loss of the ultrasonic signals used for measuring the temperature. For the 
Author Pre-Print - Journal of Applied Physics - May 2014Journal of Applied Physics 115(20):204905-204905-8 DOI: 10.1063/1.4875876

same reason it is unlikely that echoes from rays propagating in the second row will be obtained. This simulation also indicates the importance of propagating an ultrasonic beam in the central axis above a row of subassemblies to reach a subassembly head and carry out a temperature measurement. Targeting for a subassembly head without taking this condition into account with another acoustic path, or using a transducer emitting an ultrasonic beam with too large aperture, would significantly complicate the processing of the received signal.

With the third set of thermo-hydraulic data, the effect of the flow speed on the propagation of the ultrasonic waves is studied. In these simulations five rays are propagated at angles of $5^{\circ}, 2.5^{\circ}, 0^{\circ},-2.5^{\circ}$ and $-5^{\circ}$ in relation to the axis $0 x$. These rays appeared in figure FIG 9A.

A first simulation is performed in which the speed of the flow is not taken into account, and the five 5 rays meet at a point. These rays appear as crosses in figure FIG 9B. This is the centre of the arc of circle from which the rays are emitted. In this way all the rays have travelled the same distance. 

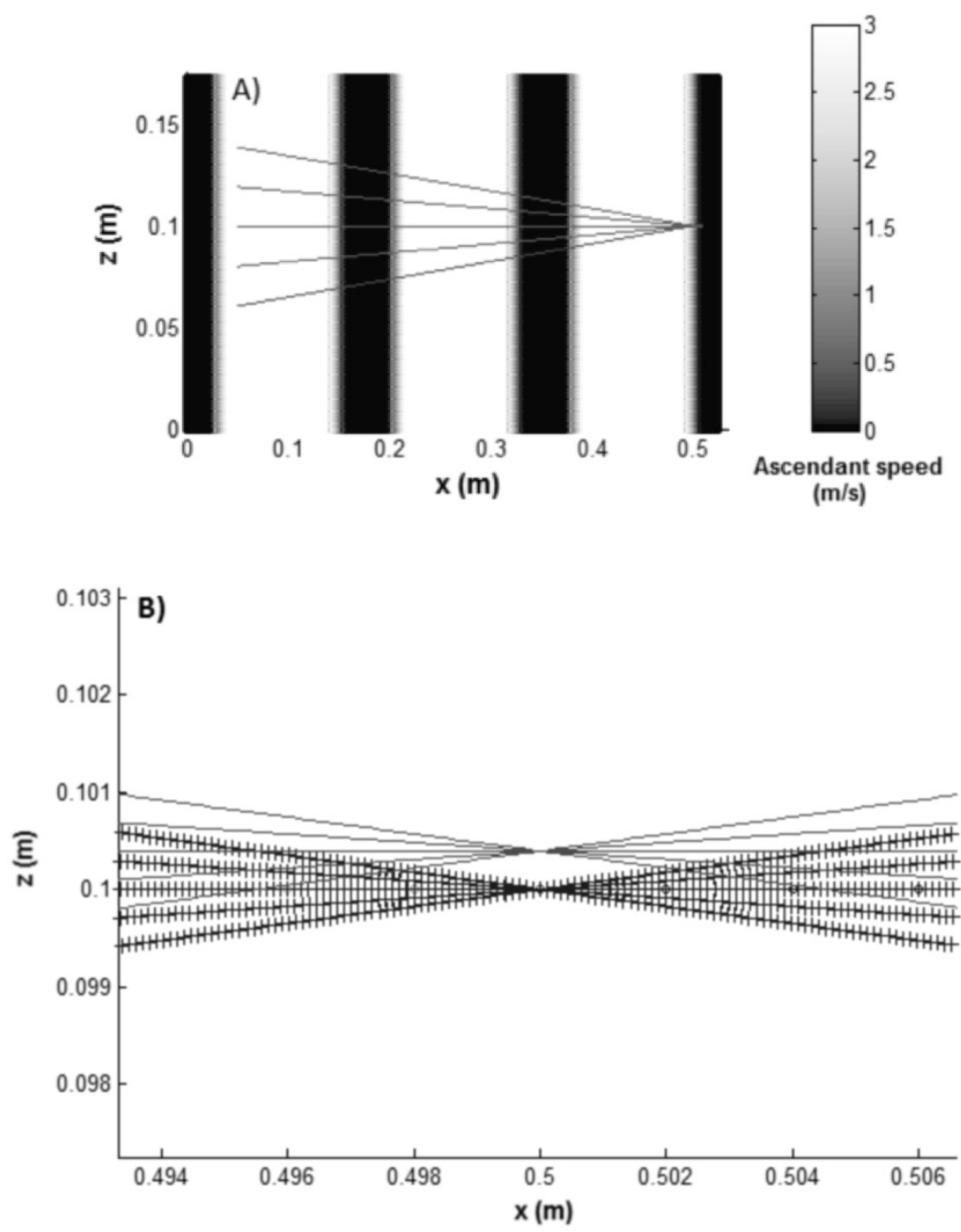

FIG 9.A) Ray propagation across ascendant flows. B) Zoom on the focusing point of the rays. Crosses: calculation of the ray path in a static medium. Line: calculation of the ray path takes into account the influence of the flow velocity.

In another simulation, the calculation of the ray path takes into account the influence of the flow velocity. Rays thus obtained are shown as lines in the figure FIG 9B. In this simulation the focusing point of the rays is highest along the axis $\mathrm{Oz}$, at a distance of about $0.5 \mathrm{~mm}$ from the precedent focusing point. All rays have been deflected by the flow of sodium following the axis Oz.

Furthermore the time of fly of each ray to reach the focus point is also different. The rays propagating with a downward angle relative to the axis Ox was slowed, and rays propagating with a bottom angle were accelerated. These results appear in the 
Author Pre-Print - Journal of Applied Physics - May 2014Journal of Applied Physics 115(20):204905-204905-8 DOI: 10.1063/1.4875876

TABLE 1.

TABLE 1. Variation in time of flight to reach the focusing point.

\begin{tabular}{|c|c|}
\hline $\begin{array}{c}\text { Propagation angle } \\
\text { in relation to axe Ox (degrees) }\end{array}$ & $\begin{array}{c}\text { Variation in time of flight } \\
\text { to reach the focusing point }(s)\end{array}$ \\
\hline-5 & $1.40 \cdot 10^{-8}$ \\
\hline-2.5 & $6.93 \cdot 10^{-9}$ \\
\hline 0 & $-9.0 \cdot 10^{-11}$ \\
\hline 2.5 & $-7.13 \cdot 10^{-9}$ \\
\hline 5 & $-1.41 \cdot 10^{-8}$ \\
\hline
\end{tabular}

These changes in the time of flight of rays come from the component of the flow velocity which is added or subtracted to the speed of ultrasound.

In the case of acoustic thermometry, where the ultrasonic beam has a downward trajectory to the sub-assembly head, then is reflected and returns with an upward trajectory to the transducer, changes in time of flight will be subtracted $^{10}$.

Moreover considering that the vertical deflection undergone by these rays is less than one millimetre, it seems that the influence of the flow velocity, in these specific thermo-hydraulic and acoustic conditions, are too minor to have a significant impact on the ultrasonic thermometry. It will however be necessary to take account of other turbulent hydraulic phenomena that exist above the core (impact of the core cover plug, turbulence near the control and command rods, etc.), before generalising this conclusion.

\section{CONCLUSION}


Author Pre-Print - Journal of Applied Physics - May 2014Journal of Applied Physics

115(20):204905-204905-8 DOI: 10.1063/1.4875876

In this study a simulation code has been implemented in order to calculate the path follow by an acoustic wave in a turbulent sodium flow and inhomogeneous temperature. The application of the method of Gaussian beams summation also enables to determine the amplitude of the wavefront of the acoustic beam at various time of propagation.

The establishment of SFR core instrumentation such as acoustic thermometry is long and complex. The results calculated by this code are thus very useful to quickly test several positions or orientations of the transducer, or assess the impact of various thermal-hydraulic parameters on the temperature measurement. The first results presented in this paper indicate that it is preferable to orientate the transducer in the axis of a row of assemblies in order to achieve a particular assembly. These results predict concentration of ultrasounds energy along the axis of that row, and thus in the area where the echoes on the edges of assemblies are produce. Furthermore under the specific case covered in this study, the influence of the velocity of flow seems to not have a significant effect on the ultrasound propagation. This code has been implemented for liquid sodium in the heart of a SFR, but it could be used for many fluids, such as water or oil, and could be used for other kind of ultrasonic instrumentation such as debitmetry or telemetry.

Finally, two experimental verifications of the model used in this code are currently being carried out. In the first one, thermal inhomogeneities are created in a specific fluid and ultrasonic waves are propagated in it. The fluid is chosen for its ability to slow the convection. First results are presented in reference 30 . The other one uses Kelvin-Helmholtz instabilities in water with a homogeneous temperature to determine the influence of turbulence on acoustic wave propagation. For these experiments, a comparison between the numerical data from the simulations and the experimental data is realised to determine the validity of the theoretical ray model. 
Author Pre-Print - Journal of Applied Physics - May 2014Journal of Applied Physics

115(20):204905-204905-8 DOI: 10.1063/1.4875876

REFERENCES

${ }^{1}$ F. Gauche, Magnetohydrodynamics 48, 191 (2012).

${ }^{2}$ F. Jadot, F. Baqué, J. P. Jeannot, G. De Dinechin, J. M. Augem, and J. Sibilo, Conf. ANIMMA (2011).

${ }^{3}$ M. Cavaro, J. Moysan, C. Payan, C. Gueudré, G. Corneloup, and F. Baqué, Proceedings of Meetings on Acoustics, 7, 045005 (2009).

${ }^{4}$ K. Paumel, J. Moysan, D. Chatain, G. Corneloup, and F. Baqué, J. Appl. Phys., 110, 044910 (2011).

${ }^{5}$ N. Massacret, J. Moysan, M. A. Ploix, J. P. Jeannot, and G. Corneloup, AIP Conference Proceedings, 1511, 1693 (2013).

${ }^{6}$ J. Daw, J. Rempe, S. Taylor, J. Crepeau, and S. C. Wilkins, Proc. NPICHMIT 2010, (2010).

${ }^{7}$ A. Baba, C. T. Searfass, and B.R. Tittmann, Applied Physics Letters, 97, 232901 (2010).

${ }^{8}$ D. Parks, S. Zhang, B. R. Tittmann, J. Nl. IEEE Transactions on UFFC, 60, 1010 (2013).

${ }^{9}$ D. A Parks, B. T. Reinhardt and B. R. Tittmann, Rev. of Progress in QNDE, AIP Conf. Proc. 1511, 1678 (2013).

${ }^{10}$ L. Leibowit, M. Chasanov, and R. Blomquis, J. Appl. Phys., 42, 2135 (1971).

${ }^{11}$ J. A. McKnight, I. D. Macleod, and E. J. Burton, Patent nº8501470 (1985).

${ }^{12}$ C. G. Taylor and S. Birch, AEA Technology, Risley, (1992).

${ }^{13}$ Y. Liu, G. Du, L. Tao and F. Shen, Journal of hydrodynamics, 23, 89 (2011).

${ }^{14}$ B. Iooss, C. Lhuillier, and H. Jeanneau, Ultrasonics, 40, 1009 (2002).

${ }^{15}$ L. Dallois, PhD, École Centrale de Lyon, (2000).

${ }^{16}$ S. Kon, K. Yamaguchi, H. Ohbayashi, Y. Tasaka, Y. Murai, and Y. Takeda, Nihon Kikai Gakkai Ryutai Kogaku Bumon Koenkai Koen Ronbunshu CD-ROM, 84, 19 (2006).

${ }^{17}$ D. Tenchine and H. Y. Nam, AIChE Symp. Ser., 83, 151 (1987).

${ }^{18}$ J. P. Moro and D. Tenchine. IAEA International Working Group on Fast Reactors, Specialist Meeting on Correlation Between Mateial Properties and Thermo-Hydraulics Conditions in LMFBRs (1994).

${ }^{19}$ J. Y. Zhang, W. J. Xu, J. Carlier, X. M. Ji, B. Nongaillard, S. Queste and Y.P. Huang, Ultrasonics, 52, 47 (2012).

${ }^{20}$ D. Komatitsch and J. Tromp, Geophys. J. Int., 139, 806 (1999).

${ }^{21}$ B Hosten, C Bacon, C Biateau, Journal of the Acoustical Society of America, 124, 3491 (2009).

${ }^{22}$ P. Moussou, P. Vaugrante, M. Guivarch, D. Seligmann, Flow Induced Vibration -International Conference, 579 (2000).

${ }^{23}$ A. D. Pierce, Acoustical Soc of America, ISBN : 0883186128, (1989).

${ }^{24}$ V. Cerveny, M. Popov, and I. Psencik, Geophys. J. R. Astron. Soc., 70, 109 (1982).

${ }^{25}$ D. Fiorina, PhD, Ecole Centrale de Lyon (1998).

${ }^{26}$ B. Lü, PhD, Université du Maine, (2012).

${ }^{27}$ V. M. Babič and V. S. Buldyrev, Alpha Science International, ISBN : 184265232X (1991).

${ }^{28}$ M. Karweit, P. Blancbenon, D. Juve, and G. Comtebellot, J. Acoust. Soc. Am., 89, 52 (1991). 
Author Pre-Print - Journal of Applied Physics - May 2014Journal of Applied Physics 115(20):204905-204905-8 DOI: 10.1063/1.4875876

${ }^{29}$ S. Moriya and I. Ohshima, Nucl. Eng. Des., 120, 385 (1990).

${ }^{30}$ N. Massacret, J. Moysan, M.A. Ploix \&J.P. Jeannot, 13th Int. Sympo. Non Destruc. Char. Mat., Le Mans, (2013). 